\title{
A Fluid-Flow Model for Backlog-Based CSMA Policies
}

\author{
Atilla Eryilmaz \\ Dept. of Electrical and \\ Computer Engineering \\ Ohio State University \\ Columbus, $\mathrm{OH}$ \\ eryilmaz@ece.osu.edu
}

\author{
Peter Marbach \\ Dept. of Computer Science \\ Univeristy of Toronto \\ Toronto, Canada \\ marbach@cs.toronto.edu
}

\author{
Asuman Ozdaglar \\ Dept. of Electrical Engineering \\ and Computer Science \\ MIT \\ Cambridge, MA \\ asuman@mit.edu
}

\begin{abstract}
We present a fluid flow model to analyze backlog-based CSMA policies. The model is obtained using a CSMA fixed point approximation that has been recently proposed and analyzed. We provide numerical case studies to illustrate the accuracy of the fluid-flow model.
\end{abstract}

\section{Keywords}

Carrier Sense Multiple Access, Fixed Point Analysis, Wireless Networks, Throughput-optimality, Backlog-based Scheduling, Distributed Algorithms

\section{INTRODUCTION}

Recently, there has been a growing interest in the development of distributed transmission policies in interferencelimited wireless networks. A key objective of these distributed policies is to achieve any throughput within the $c a-$ pacity region ${ }^{1}$ with of the network. Most recent work in this area focused on distributed mechanisms that can implement the throughput-optimal (or max-weight) policies, proposed in the seminal work of Tassiulas and Ephremides [16]. In view of their low-complexity nature, random access schemes provide an attractive alternative class of distributed transmission strategies. Despite considerable progress on the study of distributed max-weight policies (see [3, 7, 8, 12, 15, $17]$ ), there has been little formal analysis of random access schemes for networks. The random access methods studied in earlier works (e.g. $[4,6]$ ) used the slotted Aloha frame-

\footnotetext{
${ }^{*}$ The work of the first and third authors was supported by the Control- Based Mobile Ad-Hoc Networking (CBMANET) Program under DARPA subcontract no. 060786. The work of the first author was supported by the DTRA Grant HDTRA1-08-1-0016. The work of the second author was supported by a NSERC strategic grant.

${ }^{1}$ The capacity region contains the set of arrival rates that are achievable by some feasible policy. A more rigorous definition will be provided later.
}

Permission to make digital or hard copies of all or part of this work for personal or classroom use is granted without fee provided that copies are not made or distributed for profit or commercial advantage and that copies bear this notice and the full citation on the first page. To copy otherwise, to republish, to post on servers or to redistribute to lists, requires prior specific permission and/or a fee.

WICON' 08, November 17-19, 2008, Maui, Hawaii, USA.

Copyright 2008 ISCT 978-963-9799-36-3 . work with emphasis on distributed implementation and fairness rather than throughput performance.

In this paper, we focus on CSMA policies which differs from the slotted Aloha in that each transmitter detects the idle channel condition (potentially with some sensing delay) before attempting a transmission. The analysis that we use is based on a tractable fixed point formulation, called the CSMA fixed point, that was presented in [11]. This formulation allows to characterize the performance and achievable rate region of CSMA policies. In [10], the CSMA fixed point was used to analyze the performance of a distributed scheduling and rate control mechanism to achieve fairness and throughput-optimality in multihop wireless networks. The results of [10] is are based on an operating point analysis. In this paper, we use the CSMA fixed point to formulate a fluid-flow model for the dynamics of the policies presented [10] to show that for the symmetric case of homogeneous arrival rates this policy stabilizes any input arrival rate within the achievable rate region. We further provide simulations to illustrate the behavior of the stochastic system.

The paper is organized as follows: Section 3 describes the system model that we consider, and Section 5 describes CSMA policies that we use in our analysis. Section 6 provides a summary of the results presented in [11] on the CSMA fixed point approximation. Section 8 presents a dynamic queue-length based scheduling policy and study its stability properties through both analysis and simulation.

\section{RELATED WORK}

For single-hop networks where all nodes are within transmission range of each other, the performance of CSMA policies is well-understood. For the case where nodes are saturated and always have a packet to sent, the achievable rate region of CSMA policies is easily obtained [13]. For the case where nodes only make a transmission attempt when they have a packet to transmit has recently been derived for the limiting regime of many small flows [13]. Furthermore, the well-known "infinite node" approximations provides a simple characterization for the throughput of a given CSMA policy, as well as the achievable rate region of CSMA policies, in the case of a single-hop networks [2]. This approximation has been instrumental in the understanding of the performance of CSMA policies, as well as for the design of practical protocols for wireless local area networks [2]. We will briefly review this approximation in Section 6.1, and use it to formulate our CSMA fixed point approximation in Section 6.2.

For general multihop networks, results for CSMA poli- 
cies are available for idealized situation of instantaneous channel feedback. This assumption of instantaneous channel feedback allows the elimination of collisions, which significantly simplifies the analysis. Under this assumption, Jiang and Walrand in [5] derived a dynamic CSMA policy that, combined with rate control, achieves throughput-optimality while satisfying a given fairness criterion. Similar results have been independently derived by Shah and Sreevastsa in [14] in the context of optical networks. While these results are obtained for a simplified (idealized) model of CSMA policies, they suggest the fact that CSMA policies might be throughput-optimal. In this work, we confirm this suggestion in the presence of collisions. It should be noted that the result by Jiang and Walrand has been obtained for general interference models, whereas our analysis focuses on wireless networks with primary interference constraints. However, our analysis does not assume instantaneous channel feedback and hence takes packet collisions into account.

\section{SYSTEM MODEL}

We consider a fixed wireless network composed of a set $\mathcal{N}$ of nodes with cardinality $N$, and a set $\mathcal{L}$ of directed links with cardinality $L$. A directed link $(i, j) \in \mathcal{L}$ indicates that node $i$ is able to send data packets to node $j$. We assume that the rate of transmission is the same for all links and all packets are of a fixed length. Throughout the paper we rescale time such that the time it takes to transmit one packet is equal to one time unit.

For a given node $i \in \mathcal{N}$, let $\mathcal{U}_{i}:=\{j \in \mathcal{N}:(i, j) \in \mathcal{L}\}$ be the set of upstream nodes, i.e. the set containing all nodes which can receive packets from $i$. Similarly, let $\mathcal{D}_{i}:=$ $\{j \in \mathcal{N}:(j, i) \in \mathcal{L}\}$ be set of downstream nodes, i.e. the set containing all nodes $j$ from which $i$ can receive packets. Collectively, we denote the set of all the neighbors of node $i$ as $\mathcal{N}_{i}:=\mathcal{U}_{i} \cup \mathcal{D}_{i}$. Also, we let $\mathcal{L}_{i}:=\left\{(i, j): j \in \mathcal{D}_{i}\right\}$ be the set of outgoing links from node $i$, i.e. the set of all links from node $i$ to its upstream nodes $\mathcal{U}_{i}$.

Throughout the paper, we assume that $\mathcal{U}_{i}=\mathcal{D}_{i}$, for all $i \in \mathcal{N}$ so that we have $\mathcal{U}_{i}=\mathcal{D}_{i}=\mathcal{N}_{i}$, for each $i \in \mathcal{N}$. This assumption simplifies the notation as we can use a single set $\mathcal{N}_{i}$ to represent both $\mathcal{D}_{i}$ and $\mathcal{U}_{i}$. Our analysis can be extended to the more general case requiring only notational changes.

We assume that there is a fixed set of routes $\mathcal{R}$ which defines the possible traffic paths in the network. For a given route $r \in \mathcal{R}$, let $s_{r}$ be its source node and $d_{r}$ be its destination node. Furthermore, we use the convention that $r$ is given by the set of links that the route traverses, i.e. we let

$$
r=\left\{\left(s_{r}, i\right),(i, j), \cdots,(v, w),\left(w, d_{r}\right)\right\}
$$

be the set of links traversed by the route. We allow several routes to be defined for a given source and destination pair $(s, d), s, d \in \mathcal{N}$.

In the following, we describe a network by the tuple $(\mathcal{L}, \mathcal{R})$ since the set of nodes $\mathcal{N}$ can be derived from the set $\mathcal{L}$.

We focus on networks under the well-known primary interference, or node exclusive interference, model [17].

Definition 1 (Primary Interference Model). A packet transmission over link $(i, j) \in \mathcal{L}_{i}$ is successful if only if within the transmission duration there exist no other activity over any other link $(m, n) \in \mathcal{L}$ which shares a node with $(i, j)$.
The primary interference model applies, for example, to wireless systems where multiple frequencies/codes are available (using FDMA or CDMA) to avoid interference, but each node has only a single transceiver and hence can only send to or receive from one other node at any time.

We characterize the network traffic with a rate vector $\lambda:=$ $\left\{\lambda_{r}\right\}_{r \in \mathcal{R}}$ where $\lambda_{r}, \lambda_{r} \geq 0$, is the mean packet arrival rate in packets per unit time on route $r \in \mathcal{R}$.

Given the rate vector $\lambda=\left\{\lambda_{r}\right\}_{r \in \mathcal{R}}$, we let

$$
\lambda_{(i, j)}:=\sum_{r:(i, j) \in \mathcal{R}_{r}} \lambda_{r}, \quad(i, j) \in \mathcal{L},
$$

be the mean packet arrival rate to link $(i, j)$. Similarly, we let

$$
\Lambda_{i}:=\sum_{j \in \mathcal{N}_{i}}\left[\lambda_{(i, j)}+\lambda_{(j, i)}\right], \quad i \in \mathcal{N} .
$$

be the mean packet arrival rate to node $i \in \mathcal{N}$.

With each route $r$ we associate a utility function $U_{r}$ that depends on the rate $\lambda_{r}$ allocated to route $r$. We make the following assumption.

Assumption 1. The utility function $U_{r}\left(\lambda_{r}\right), r \in \mathcal{R}$, is increasing and strictly concave with $U_{r}(0)=0$ and $\lim _{\lambda_{r} \rightarrow \infty} U_{r}^{\prime}\left(\lambda_{r}\right)=0$.

\section{CAPACITY REGION}

Consider a fixed network $(\mathcal{L}, \mathcal{R})$ with traffic vector $\lambda=$ $\left\{\lambda_{r}\right\}_{r \in \mathcal{R}}$. A scheduling policies $\pi$ then defines the rules that are used to schedule packet transmissions on each link $(i, j) \in \mathcal{L}$. In the following we focus on policies $\pi$ that have a well-defined link service rates as a function of the rate vector $\lambda=\left\{\lambda_{r}\right\}_{r \in \mathcal{R}}$.

DEFINITION 2. (Service Rate): Consider a fixed network $(\mathcal{L}, \mathcal{R})$. The link service rate $\mu_{(i, j)}^{\pi}(\lambda),(i, j) \in \mathcal{L}$, of policy $\pi$ for the traffic vector $\lambda=\left\{\lambda_{r}\right\}_{r \in \mathcal{R}}$ is the fraction of time node $i$ spends successfully transmitting packets on link $(i, j)$ under $\pi$ and $\lambda$, i.e. the fraction of time node $i$ sends packets over link $(i, j)$ that do not experience a collision.

Let $\mathcal{P}$ be the class of all policies $\pi$ that have well-defined link service rates. Note that this class contains a broad range of scheduling policies, including dynamic policies such as queue-length-based policies [16], as well as noncausal policies that know the future arrival of the flows. We then define network stability as follows.

Definition 3 (Stability). For a given network $(\mathcal{L}, \mathcal{R})$, let $\mu^{\pi}(\lambda)=\left\{\mu_{(i, j)}^{\pi}(\lambda)\right\}_{(i, j) \in \mathcal{L}}$ the link service rates of policy $\pi, \pi \in \mathcal{P}$, for the rate vector $\lambda=\left\{\lambda_{r}\right\}_{r \in \mathcal{R}}$. We say that policy $\pi$ stabilizes the network for $\lambda$ if

$$
\lambda_{(i, j)}<\mu_{(i, j)}^{\pi}(\lambda), \quad \text { for all }(i, j) \in \mathcal{L} .
$$

This commonly used stability criteria [16] requires that for each link $(i, j)$ the link service rate $\mu_{(i, j)}^{\pi}(\lambda)$ is larger than the arrival rate $\lambda_{(i, j)}$. The capacity region of a network $(\mathcal{L}, \mathcal{R})$ is then defined as follows.

DeFinition 4. (Capacity Region) For a given a network $(\mathcal{L}, \mathcal{R})$, the capacity region $\mathcal{C}$ is equal to the set of all traffic vectors $\lambda=\left\{\lambda_{r}\right\}_{r \in \mathcal{R}}$ such that there exists a policy $\pi \in \mathcal{P}$ that stabilizes the network for $\lambda$, i.e. we have

$\mathcal{C}=\left\{\lambda \geq 0: \exists \pi \in \mathcal{P}\right.$ with $\left.\lambda_{(i, j)}<\mu_{(i, j)}^{\pi}(\lambda), \forall(i, j) \in \mathcal{L}\right\}$ 


\section{CSMA SCHEDULING POLICIES}

In the following, we study CSMA policies as defined below.

\subsection{CSMA Policies}

We consider CSMA policies that are given by a transmission attempt probability vector $\mathbf{p}=\left(p_{(i, j)}\right)_{(i, j) \in \mathcal{L}} \in[0,1]^{L}$ and a sensing period (or idle period) $\beta>0$. The policy works as follows: each node, say $i$, senses the activity on its outgoing links $l \in \mathcal{L}_{i}$. If link $(i, j) \in \mathcal{L}_{i}$ has been idle for a duration of an idle period $\beta$, i.e. node $i$ and $j$ have not sent, or received, a packet for $\beta$ time units, then $i$ starts a transmission of a single packet on link $(i, j)$ with probability $p_{(i, j)}$, independent of all other events in the network. If node $i$ does not start a packet transmission, then link $(i, j)$ has to remain idle for another period of $\beta$ time units before $i$ again has the chance to start a packet transmission. Thus, the epochs at which node $i$ has the chance to transmit a packet on link $(i, j)$ are separated by periods of length $\beta$ during which link $(i, j)$ is idle, and the probability that $i$ starts a transmission on link $(i, j)$ after the link has been idle for $\beta$ time units is equal to $p_{(i, j)}$,

We assume that packet transmission attempts are made according to above description regardless of the availability of packets at the transmitter. In the event of a transmission decision in the absence of packets, the transmitting node transmits a dummy packet, which is discarded at the receiving end of the transmission.

The duration of an idle period $\beta$ is again given relative to the length of a packet transmission which is assumed to take one unit time, i.e. if the length of an sensing period is $L_{i}$ seconds and the length of a packet transmission is $L_{p}$ seconds, then we have $\beta=L_{i} / L_{p}$. For a fixed $L_{i}$, the duration of an idle period $\beta$ will become small if we increase the packet lengths, and hence the packet transmission delay $L_{p}$.

Given the length of an sensing period $\beta$, in the following we will sometimes refer to $\mathbf{p}$ as the CSMA policy.

\subsection{Achievable Rate Region of CSMA Policies}

One can show [11] that a CSMA policy $\mathbf{p}$ has a welldefined link service rate vector, i.e. CSMA policies are contained in the set $\mathcal{P}$. Given a sensing period $\beta$, we denote with $\mu(\mathbf{p})=\left\{\mu_{(i, j)}\right\}_{(i, j) \in \mathcal{L}}$ the link service rate vector under the CSMA policy $\mathbf{p}$. Note that for a given $\beta$, the link service rate under a CSMA policy depend only on the transmission attempt probability vector $\mathbf{p}$, but not on the arrival rates $\lambda$. The achievable rate region of CSMA policies is then given as follows.

Definition 5 (Achievable Rate Region). For a given network $(\mathcal{L}, \mathcal{R})$ and a given sensing period $\beta$, the achievable rate region of CSMA policies is given by the set of rate vectors $\lambda=\left\{\lambda_{r}\right\}_{r \in \mathcal{R}}$ for which there exists a CSMA policy $\mathbf{p}$ that stabilizes the network for $\lambda$, i.e. we have that $\lambda_{(i, j)}<\mu_{(i, j)}(\mathbf{p}),(i, j) \in \mathcal{L}$.

Let $\mathcal{C}_{C S M A}$ be the achievable rate region of CSMA policies.

In [11], it was shown that the achievable rate region $\mathcal{C}_{C S M A}$ of CSMA policies is equal to the capacity region $\mathcal{C}$ under the limiting regime of large networks with many small flows and a small sensing period $\beta$. This result was obtained using a novel CSMA fixed point approximation that extends the well-known infinite node approximation for single-hop networks to the case of multihop networks. In the next section, we provide a summary of the results in [11].

\section{CSMA FIXED POINT APPROXIMATION}

In this section, we present the CSMA fixed point approximation of [11], where we first briefly review the infinite node approximation for single-hop networks. In the following we will use $\tau$ to denote the services rates obtained under our analytical formulations that we use to approximate the actual service rates $\mu$ under a CSMA policy as defined in Section 5 .

\subsection{Infinite Node Approximation for Single- Hop Networks}

Consider a single-hop network where $N$ nodes share a single communication channel, i.e. where nodes are all within transmission range of each other. In this case, a CSMA policy is given by the vector $\mathbf{p}=\left(p_{1}, \cdots, p_{N}\right) \in[0,1]^{N}$ where $p_{n}$ is the probability that node $n$ starts a packet transmission after an idle period of length $\beta$ [2].

The network throughput, i.e. the fraction of time the channel is used to transmit packets that do not experience a collision, can then be approximated by (see for example [2])

$$
\tau(G(\mathbf{p}))=\frac{G(\mathbf{p}) e^{-G(\mathbf{p})}}{\beta+1-e^{-G(\mathbf{p})}}
$$

where $G(\mathbf{p})=\sum_{n=1}^{N} p_{n}$. Note that $G(\mathbf{p})$ captures the expected number of transmissions attempt after an sensing period $\beta$ for a given CSMA policy $\mathbf{p}$.

The following results are well-known. For $\beta>0$, we have that $\tau(G)<1, G \geq 0$, and for $G^{+}(\beta)=\sqrt{2 \beta}, \beta>0$, we have that $\lim _{\beta \downarrow 0} \tau\left(G^{+}(\beta)\right)=1$.

Using (3), the service rate $\mu_{n}(\mathbf{p})$ of node $n$ under a given static CSMA policy $\mathbf{p}$ can be approximated by

$$
\tau_{n}(\mathbf{p})=\frac{p_{n} e^{-G(\mathbf{p})}}{1+\beta-e^{-G(\mathbf{p})}}, \quad n=1, \ldots, N .
$$

In the above expression, $p_{n}$ is the probability that node $n$ starts a packet transmission if the channel has been idle for a sensing period of duration $\beta$, and $e^{-G(\mathbf{p})}$ characterizes the probability that this attempt is successful, i.e. the attempt does not collide with an attempt by any other node.

Similarly, the fraction of time that the channel is idle can be approximated by

$$
\rho(\mathbf{p})=\rho(G(\mathbf{p}))=\frac{\beta}{\beta+1-e^{-G(\mathbf{p})}},
$$

where we have that $\lim _{\beta \downarrow 0} \rho\left(G^{+}(\beta)\right)=0$.

\subsection{CSMA Fixed Point Approximation for Mul- tihop Networks}

We extend the above approximation for single-hop networks to multihop networks as follows.

For a given a sensing period $\beta$, we approximate the fraction of time $\rho_{i}(\mathbf{p})$ that node $i$ is idle under the CSMA policy $\mathbf{p}$ by the following fixed point equation,

$$
\rho_{i}(\mathbf{p})=\frac{\beta}{\beta+1-e^{-G_{i}(\mathbf{p})}}, \quad i=1, \cdots, N,
$$

where

$$
G_{i}(\mathbf{p})=\sum_{j \in \mathcal{N}_{i}}\left[p_{(i, j)}+p_{(j, i)}\right] \rho_{j}(\mathbf{p}), \quad i=1, \cdots, N
$$

which approximately measures the transmission attempt rate of node $i$ given that it is idle. We refer to the above fixed point equation as the CSMA fixed point equation, and to a 
solution $\rho(\mathbf{p})=\left(\rho_{1}(\mathbf{p}), \cdots, \rho_{N}(\mathbf{p})\right)$ and $G(\mathbf{p})=\left(G_{1}(\mathbf{p}), \cdots, G_{N}(\mathbf{p})\right.$ inder the CSMA fixed point approximation contains the set to the fixed point equation as a CSMA fixed point.

The intuition behind the CSMA fixed point equation Eq. (6) and Eq. (7) is as follows: suppose that the fraction of time that node $i$ is idle under the static CSMA policy $\mathbf{p}$ is equal to $\rho_{i}(\mathbf{p})$, and suppose that the times when node $i$ is idle are independent of the processes at all other nodes. If node $i$ has been idle for $\beta$ time units, i.e. node $i$ has not received or transmitted a packet for $\beta$ time units, then node $i$ can start a transmission attempt on link $(i, j), j \in \mathcal{N}_{i}$, only if node $j$ also has been idle for an idle period of $\beta$ time units. Under the above independence assumption, this will be (roughly) the case with probability $\rho_{j}(\mathbf{p})$, and the probability that node $i$ start a packet transmission on the link $(i, j), j \in \mathcal{N}_{i}$, given that it has been idle for $\beta$ time units is (roughly) equal to $p_{(i, j)} \rho_{j}(\mathbf{p})$. Similarly, the probability that node $j \in \mathcal{N}_{i}$ starts a packet transmission on the link $(j, i)$ after node $i$ has been idle for $\beta$ time units is (roughly) equal to $p_{(j, i)} \rho_{j}(\mathbf{p})$. Hence, the expected number of transmission attempts that node $i$ makes or receives, after it has been idle for $\beta$ time units is (roughly) given by Eq. (7). Using Eq. (5) of Section 6.1 , the fraction of time that node $i$ is idle under $\mathbf{p}$ can then be approximated by Eq. (6).

For a given an sensing period $\beta$, we can then use the CSMA fixed point $G(\mathbf{p})$ for a policy $\mathbf{p}$ to approximate the link service rate $\mu_{(i, j)}(\mathbf{p})$ under a CSMA policy $\mathbf{p}$ by

$$
\tau_{(i, j)}(\mathbf{p})=\frac{p_{(i, j)} \rho_{j}(\mathbf{p}) e^{-G_{i}(\mathbf{p})}}{1+\beta-e^{-G_{i}(\mathbf{p})}} e^{-G_{j}(\mathbf{p})}, \quad(i, j) \in \mathcal{L} .
$$

Note that the above equation is similar to (4) where $p_{(i, j)} \rho_{j}(\mathbf{p})$ captures the probability that node $i$ starts a packet transmission on link $(i, j)$ if node $i$ has been idle for $\beta$ time units, and $\exp \left[-\left(G_{i}(\mathbf{p})+G_{j}(\mathbf{p})\right)\right]$ is the probability that this attempt is successful, i.e. the attempt does not overlap with an attempt by any other node to capture a link that has an endpoint in common with link $(i, j)$.

There are two important questions regarding the CSMA fixed point approximation. First, one needs to show that the CSMA fixed point is well-defined, i.e. that there always exists a unique CSMA fixed point. In [11] it was shown that this is indeed the case. Second, one would like to know how accurate the CSMA fixed point approximation is. In [11] it was shown that the CSMA fixed point approximation is asymptotically accurate for large networks with many small flows and a small sensing period $\beta$.

\subsection{Approximate Achievable Rate Region}

In this section we use the CSMA fixed point approximation to characterize the achievable rate region of CSMA policies.

Consider a network $(\mathcal{L}, \mathcal{R})$ with sensing time $\beta>0$ as described in Section 3, and let $\Gamma(\beta)$ be given by

$\Gamma(\beta)=\left\{\lambda=\left\{\lambda_{r}\right\}_{r \in \mathcal{R}} \mid \Lambda_{i}<\tau\left(G^{+}(\beta)\right) e^{-\left(G^{+}(\beta)\right)}, i=1, \cdots, N\right\}$,

where $G^{+}(\beta)=\sqrt{2 \beta}$ and $\tau\left(G^{+}(\beta)\right.$ are as defined in Section 6.1 , and

$$
\Lambda_{i}=\sum_{j \in \mathcal{N}_{i}}\left[\lambda_{(i, j)}+\lambda_{(j, i)}\right], \quad i \in \mathcal{N}
$$

is as defined in Section 3.

The next result states that for a network $(\mathcal{L}, \mathcal{R})$ with sensing time $\beta>0$ the achievable rate region of CSMA policies

$\Gamma(\beta)$.

THEOREM 1. Given a network $(\mathcal{L}, \mathcal{R})$ with sensing time $\beta>0$, for every $\lambda \in \Gamma(\beta)$ there exists a CSMA policy $\mathbf{p}$ such that

$$
\lambda_{(i, j)}<\tau_{(i, j)}(\mathbf{p}), \quad(i, j) \in \mathcal{L},
$$

where $\tau_{(i, j)}(\mathbf{p})$ is the service rate for link $(i, j)$ under the CSMA fixed point approximation as given by Eq. (8).

We refer to [11] for a proof of Theorem 1. The proof of Theorem 1 given in [11] is constructive in the sense that given a rate vector $\lambda \in \Gamma(\beta)$, it constructs a CSMA policy p such that $\lambda_{(i, j)}<\tau_{(i, j)}(\mathbf{p}),(i, j) \in \mathcal{L}$. We will use this result in the next section.

Note that from Section 6.1, we have that

$$
\lim _{\beta \rightarrow 0} G^{+}(\beta)=0
$$

and

$$
\lim _{\beta \rightarrow 0} \tau\left(G^{+}(\beta)\right)=1
$$

Using these results, we obtain that

$$
\lim _{\beta \downarrow 0} \Gamma(\beta)=\left\{\lambda=\left\{\lambda_{r}\right\}_{r \in \mathcal{R}} \mid \Lambda_{i}<1 \quad i=1, \cdots, N\right\} .
$$

Furthermore, note that any rate vector $\lambda$ for which there exists a node $i$ with $\Lambda_{i} \geq 1$ cannot be stabilized, as the service rate at each node is upper-bounded by 1 . Hence, the above result suggests that for network with a small sensing time the achievable rate region of static CSMA policies is equal to the capacity region. In [11] it was shown that this is indeed true (i.e. the characterization of the achievable rate region using the CSMA fixed point approximation is accurate) for the limiting regime of large networks with many small flows and a small sensing period $\beta$.

\section{A DISTRIBUTED CSMA MECHANISM}

In [10], a backlog-based CSMA mechanism was proposed that aims at dynamically adapting to any rate vector $\lambda$ within $\Gamma(\beta)$. However, the analysis presented in [10] was limited to an operating point analysis which does not take into account the dynamic behavior of the proposed policy. Here, we propose fluid-flow model to analyze the dynamic behavior of the policy. We derive a convergence result for a special case of a network topology and configuration that we describe below.

For our analysis, we focus on the network topology given by Fig. 1. The network consists of a set set of $N$ sender nodes $\mathcal{N}_{S}=\{1, \ldots, N\}$, and a set of $N$ receiver nodes $\mathcal{N}_{R}=$ $\{N+1, \ldots, 2 N\}$. The set of links $\mathcal{L}$ consists of all directed links $(i, j)$ from a sender $i \in \mathcal{N}_{S}$ to a receiver $j \in \mathcal{N}_{R}$. Furthermore, we have the the set of routes is equal to the set of links, i.e. we have $\mathcal{R}=\mathcal{L}$.

For this network, a CSMA policy $\mathbf{p}=\left(p_{(i, j)}\right)_{(i, j) \in \mathcal{L}} \in$ $[0,1]^{L}$ determines the probabilities $p_{(i, j)}$ with which sender $i \in \mathcal{N}_{S}$ starts a transmission of a packet to receiver $j \in \mathcal{N}_{R}$, after link $(i, j)$ has been sensed to be idle for sensing period of $\beta$ time units. Given a sensing period $\beta$, the CSMA fixed point for a policy $\mathbf{p}$ is then given by

$$
\rho_{i}(\mathbf{p})=\frac{\beta}{\left(\beta+1-e^{-G_{i}(\mathbf{p})}\right)}, \quad i=1, \cdots, 2 N,
$$




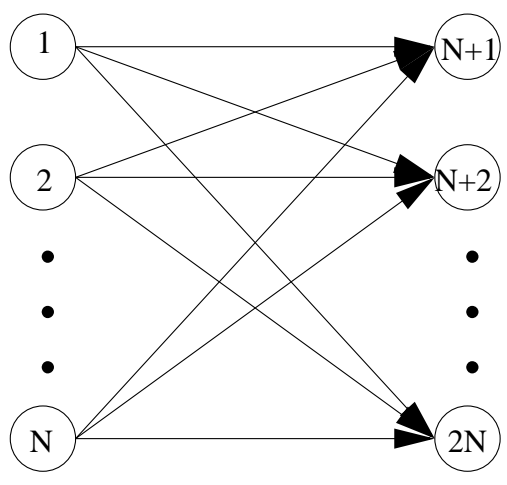

Figure 1: Network Topology consisting of $N$ sender and receiver nodes.

where

$$
G_{i}(\mathbf{p})=\sum_{j \in \mathcal{N}_{R}} p_{(i, j)} \rho_{j}(\mathbf{p}), \quad i \in \mathcal{N}_{S}
$$

and

$$
G_{j}(\mathbf{p})=\sum_{i \in \mathcal{N}_{S}} p_{(i, j)} \rho_{j}(\mathbf{p}), \quad j \in \mathcal{N}_{R}
$$

\section{A BACKLOG-BASED CSMA POLICY}

We consider the following backlog-based CSMA policy (see also [10] for a more detailed derivation and description). Whenever node $i$ detects that link $(i, j) \in \mathcal{L}$ is idle, i.e. that both node $i$ and $j$ have been idle for a sensing period of duration $\beta$, it starts a packet transmission on link $(i, j)$ with probability

$$
p_{(i, j)}=\min \left\{1-\delta, \epsilon q_{(i, j)}\right\},
$$

where $0 \leq \delta \ll 1$ and $0<\epsilon \ll 1$ are constants, and $q_{(i, j)}$ is the number of backlogged packets, or queue-length, of link $(i, j)$ at the time of transmission.

The intuition behind this policy is that links with a high input rate $\lambda_{(i, j)}$ will tend to have a larger queue-length $q_{(i, j)}$ and hence will be scheduled more often than competing connections with a smaller input rate.

One attractive feature of the above policy is its completely distributed and local nature since every node requires only to know the queue-length that originate at the node.

To understand the dynamics of the above CSMA policy, we assume that the system operates in the mode where $\epsilon q_{(i, j)}<(1-\delta)$ for all $(i, j) \in \mathcal{L}$. In Section 8.3, we will extend our arguments to the general mode of operation.

Assuming that queues are initially empty, we study in the following whether the above backlog-based CSMA policy can indeed support any input rate vector $\lambda$ that lies within the achievable rate region $\Gamma(\beta)$. In our analysis we will focus on symmetric link arrivals, i.e. $\lambda_{(i, j)}$ equal for all $(i, j) \in \mathcal{L}$. The motivation for this is twofold; not only is the analysis simpler for symmetric link arrivals, but it also intuitively represents a worst case scenario. To see this, consider any $\lambda \in \Gamma(\beta)$ and let

$$
\Lambda^{\max }(\lambda) \triangleq \max _{i \in\{1, \ldots, 2 N\}} \Lambda_{i}(\lambda) .
$$

Then, define a new arrival rate vector, $\bar{\lambda}$, with symmetric link arrivals $\bar{\lambda}_{(i, j)}=\Lambda^{\max }(\lambda) / N^{2}$ for all $(i, j)$. This im- plies that $\Lambda_{i}(\bar{\lambda})=\Lambda^{\max }(\lambda), i \in\{1, \ldots, 2 N\}$. If the backlogbased policy is able to stabilize the symmetric link arrivals with port rates $\Lambda_{i}(\bar{\lambda})=\Lambda^{\max }(\lambda), i \in\{1, \ldots, 2 N\}$, then it should also be able to stabilize input rates $\Lambda_{i}(\lambda) \leq \Lambda^{\max }(\lambda)$, $i \in\{1, \ldots, 2 N\}$. This observation suggests that symmetric link arrivals provide the worst-case scenario over all possible input rates for which the maximal port rates are the same. We provide in Subsection 8.1 a numerical case study to illustrate this result.

\subsection{Fluid-Flow Analysis}

In this section, we study the backlog-based CSMA policy for the case of symmetric link arrivals. Under our fluid-flow model, the following differential equation characterizes the evolution of the backlog at each link $(i, j) \in \mathcal{L}$.

$$
\dot{q}_{(i, j)}(t)=\left(\lambda-\tau_{(i, j)}(\mathbf{p}(t))\right)_{q_{(i, j)}(t) \geq 0},
$$

where $\mathbf{p}(t)=\epsilon \mathbf{q}(t)$ and $\tau_{(i, j)}(\mathbf{p})$ is as defined in Eq. (8). In the evolution, we use the notation: $(y)_{q \geq 0}=\max (0, y)$ if $q=0$, and $(y)_{q \geq 0}=y$ if $q>0$. This is included to guarantee that the queue-lengths never become negative.

The next lemma states that if the queue-lengths are initially empty, then the backlog $q_{(i, j)}(t)$ at all links $(i, j)$, and the CSMA fixed point $\rho_{i}(t)$ of all nodes $i$, will be equal for $t \geq 0$.

LEMMA 1. Under symmetric link arrivals, if the system is initially empty, i.e. if $\mathbf{p}(0)=\mathbf{0}$, then we have, for all $t \geq 0$, that

$$
\begin{aligned}
q_{(i, j)}(t) & =q(t), \quad \forall(i, j) \in \mathcal{L}, \\
\rho_{i}(t) & =\rho(t), \quad \forall i \in \mathcal{N} .
\end{aligned}
$$

The above results follows immediately from the fact that we consider symmetric link arrivals for which all queues are initially empty and we omit here a detailed derivation. Using the above lemma, we obtain the following result.

Proposition 1. Suppose that $N \lambda \leq \tau\left(G^{+}(\beta)\right) e^{-G^{+}(\beta)}$, i.e. the arrival vector lies in $\Gamma(\beta)$, and that the system is initially empty, i.e. $\mathbf{p}(0)=\mathbf{0}$. Then the backlog function $q(t)$ is bounded and we have

$$
\lim _{t \rightarrow \infty} N \epsilon q(t) \rho(t)=G^{*}
$$

where

$$
G^{*}=\min \left\{G \geq 0 \mid \tau(G) e^{-G}=N \lambda\right\} .
$$

We provide a proof of this proposition in Appendix A.

The above result states that the fluid-flow system is stable under the above queue-length based scheduler if the arrival rate $\lambda$ is within $\Gamma(\beta)$. Note however that this is a weak notion of stability as (a) it is based on a fluid-flow analysis and (b) we assume that the system is initialized at a "good" state, i.e. all queues are initially empty. Indeed, one can show the system will not be stable if the stochastic nature of the queue-length process is taken into account, i.e. for any $\lambda>0$ the underlying Markov chain will be transient. To address this issue we consider in Section 8.3 an AQM mechanism to stabilize the queue-lengths.

\subsection{Simulations for the Fluid-Flow model}

In this section, we present simulations of the fluid model for both symmetric and asymmetric link arrivals to support 


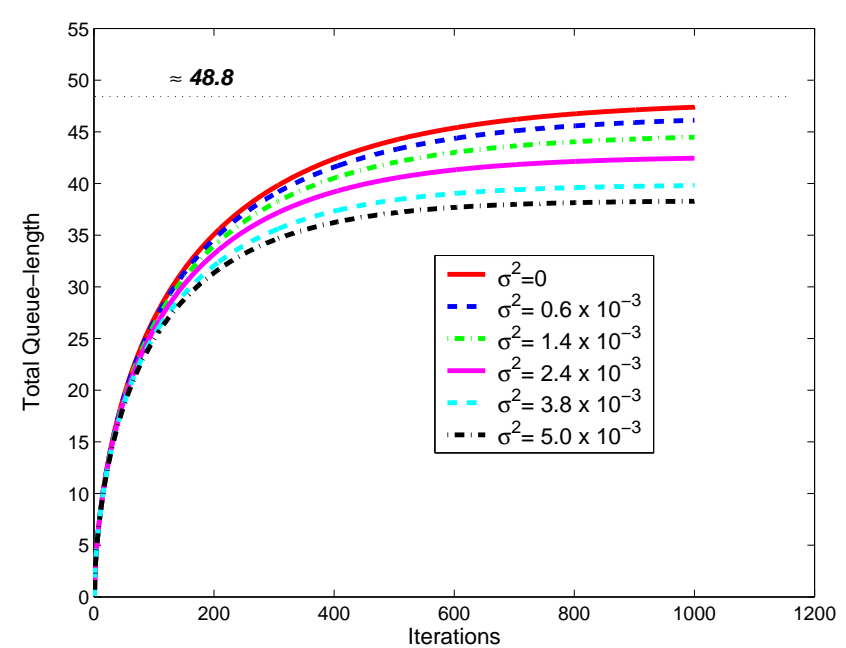

Figure 2: The total backlog evolution of the fluid model for symmetric and asymmetric link arrivals.

the theoretical arguments of Section 8.1 and to show that symmetric link arrivals works as a worst case scenario.

A typical behavior is depicted in Figure 2 for the system parameters: $N=3, \epsilon=0.003, \beta=0.001$. In the figure, $\sigma^{2}$ is the squared sum of the difference (or deviation) between the asymmetric link arrival rates and symmetric link arrival rates, and it serves as a measure of asymmetry of the arrival rate vector.

Notice that the largest achievable node throughput for $\beta=0.001$ is $\tau\left(G^{+}(\beta)\right) e^{-G^{+}(\beta)}=0.9141$. We set the symmetric link arrival rates to 0.2875 , which yields a rate of 0.8571 per node. To compare with the asymmetric case, we keep the per node rate fixed, and allow the link rates to deviate with different $\sigma^{2}$. We observe in the figure that as $\sigma^{2}$ increases the total backlog level decreases. These results support our arguments in Section 8.1.

\subsection{Active Queue Management (AQM)}

The above fluid-flow analysis indicates that the backlogbased CSMA policy is able to stabilize the network under the idealized scenario where the backlogs evolve according to the fluid-flow dynamics. Of course, the actual evolution of the queue-lengths will deviate from the fluid-flow dynamics which can potentially lead to a large surge in the queue-lengths. To prevent this, we consider the following active queue management (AQM) mechanism to stabilize the queue-lengths (see also [10] for a more detailed derivation and description).

Suppose that each link $i \in \mathcal{N}$ maintains a congestion signal $u_{i}$ which is updated at the end of each idle or busy (either a success or a collision) period according to:

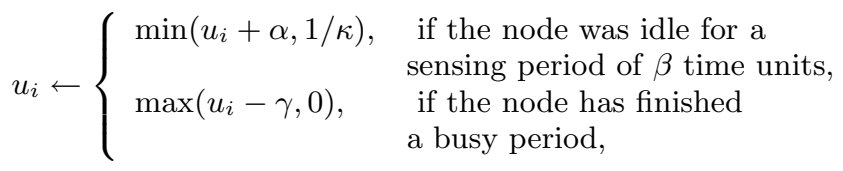

where $\alpha, \gamma$ and $\kappa$ are positive design parameters. Congestion signals are then used to determine the following dropping probabilities of packets that arrive at link $(i, j)$ :

$$
d_{(i, j)}=\min \left(\kappa\left(u_{i}+u_{j}\right), 1\right), \quad \forall(i, j) \in \mathcal{L} .
$$

In particular, each incoming packet to link $(i, j)$ is dropped with probability $d_{(i, j)}$.

The intuition behind the AQM mechanism is as follows. Each link will monitor its local activity. If too many collisions occur at link $i$, then this is interpreted as an indication that the backlog of links that pass through node $i$ are becoming too large, i.e. transmission attempt probabilities of the links that pass through node $i$ are too high. These links are then required to drop a (small) fraction of the newly arriving packets in order to prevent a further increase in the backlog.

The parameters $\alpha$ and $\gamma$ of the AQM mechanism are chosen such that

$$
G^{+}(\beta)=\ln \left(\frac{\gamma}{\gamma-\alpha}\right)
$$

in order to maximize the achieved throughput of the system. The $\kappa$ factor determines the responsiveness of the AQM mechanism to congestion: the larger it is, the more responsive is the mechanism.

One can show that the expected change in the signal $u_{i}$ between two idle periods of length $\beta$ is given by

$$
\Delta u_{i}=-\alpha+\beta\left(1-e^{-G_{i}}\right)
$$

where $G_{i}$ is the offered load at link $i$. Note that for $G_{i}=$ $G^{+}(\beta)$, the expected change in the congestion signal $u_{i}$ is equal to 0 , i.e. we have

$$
-\alpha+\gamma\left(1-e^{-G_{i}}\right)=0 .
$$

Furthermore, it can be shown that if the offered load $G_{i}$ is smaller than $G^{+}(\beta)$ then the congestion signal $u_{i}$ tends to decrease (and hence the packet-drop probability tends to decrease), whereas for $G>G^{+}(\beta)$ the congestion signal $u_{i}$ tends to increase. It follows that the packet-drop probability for connections passing through link $i$ will increase whenever $G_{i}$ exceeds $G^{+}(\beta)$. Hence, the above AQM mechanism aims at stabilizing the queue-lengths by keeping the offered load at all links below $G^{+}(\beta)$

We combine the backlog-based CSMA policy with the above AQM mechanism in order to achieve high throughput and avoid too many collisions. Such a joint algorithm was first introduced in $[9,18]$ for the case of a wireless local area network and was shown to be stable in the sense that the underlying Markov chain is positive recurrent. Here, we extend the algorithm description to incorporate the coupling between links that share a node.

\subsection{Simulations and Numerical Case Studies}

In this section, we study the behavior and performance of the backlog-based CSMA policy with AQM through simulations and numerical computations. Our goals are: to understand whether the joint algorithm stabilizes the system for any arrival rate vector within $\Gamma(\beta)$ of Section 6.3 ; and to see whether high throughput can be maintained due to AQM even when the arrival rate vector is outside the achievable region $\Gamma(\beta)$.

Unless stated otherwise, we simulate the case for $N=10$, $\beta=0.05, \kappa=0.05, \epsilon=0.01, \delta=0.05$, and $\alpha, \gamma$ of the AQM is chosen to satisfy $\alpha=\gamma\left(1-e^{-G^{+}(\beta)}\right)$.

We first test the accuracy of our fluid analysis against the behavior of the actual stochastic system under the operation of the backlog-based CSMA policy with AQM. Figure 3 depicts symmetric link arrivals of Poisson arrivals with rate 


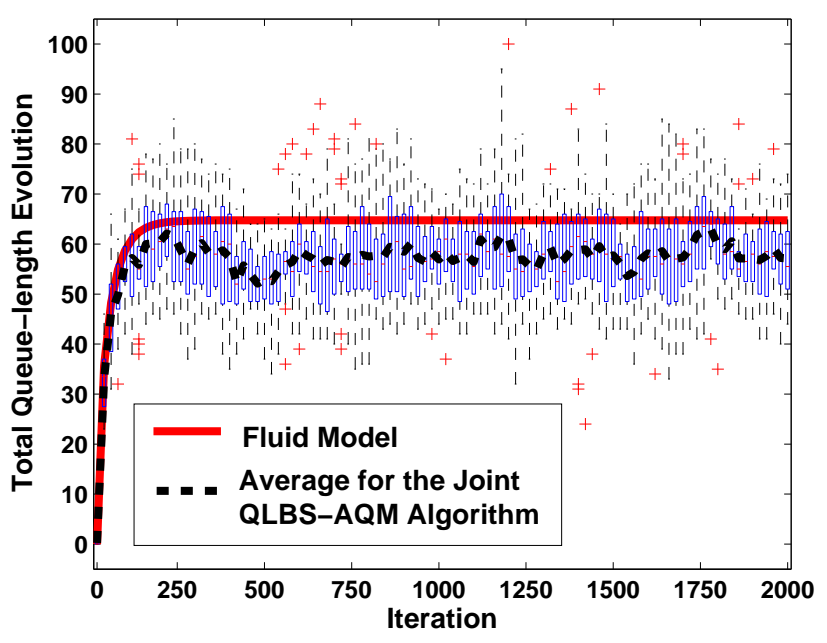

Figure 3: A typical queue-length evolution of the fluid model and the actual stochastic network under the backlog-based CSMA policy with AQM (denoted QLBS-AQM mechanism in the figure) for symmetric link arrivals with $\lambda_{(i, j)}=0.04$ packets/unit time for each link $(i, j)$.

0.4 packets/unit time per port. We observe that the queuelength evolution of the actual system closely follows the evolution of the fluid model with random fluctuations around it. The minimum, lower quartile, median, upper quartile, and maximum values of the fluctuations are also illustrated in the figure. These statistics show that the variation around the mean is not significant and the network is stable.

Figure 4 depicts the average total queue-length levels of both the fluid model and the backlog-based CSMA policy with AQM under increasing rate of arrivals for $\beta=0.05$ and 0.001. We make two observations:

- First, we note that for $\beta=0.05$ and 0.001 , the region $\Gamma(\beta)$ implies that the queues can be stabilized for link arrival rates up to 0.53 and 0.914 , respectively. This prediction is confirmed in Figure 4, where we note that the fluid model is stable until the port arrival rate level exceeds the threshold levels of 0.58 and 0.92 for $\beta=$ 0.05 and 0.001 , respectively. These values indicate the boundary of the actual achievable rate region of our algorithm while $\Gamma(\beta)$ is an inner bound. Here, we see that $\Gamma(\beta)$ is a good estimate of the actual achievable rate region with increasing accuracy as $\beta$ gets smaller.

- Second, we note that for both values of $\beta$, the stochastic system under our dynamic joint algorithm closely follows the fluid approximation for a large range of arrival rates. However, as the arrival rates approach the threshold level, AQM kicks in, resulting in increasing frequency of packet drops. This helps the system stabilize and maintain finite queue-lengths even beyond the threshold, as observed in Figure 4. Also note that low level of queue-lengths does not only imply less collisions, but also low level of queueing delay.

An important parameter of interest for any scheduling algorithm is its achieved throughput. In Figure 5, we plot

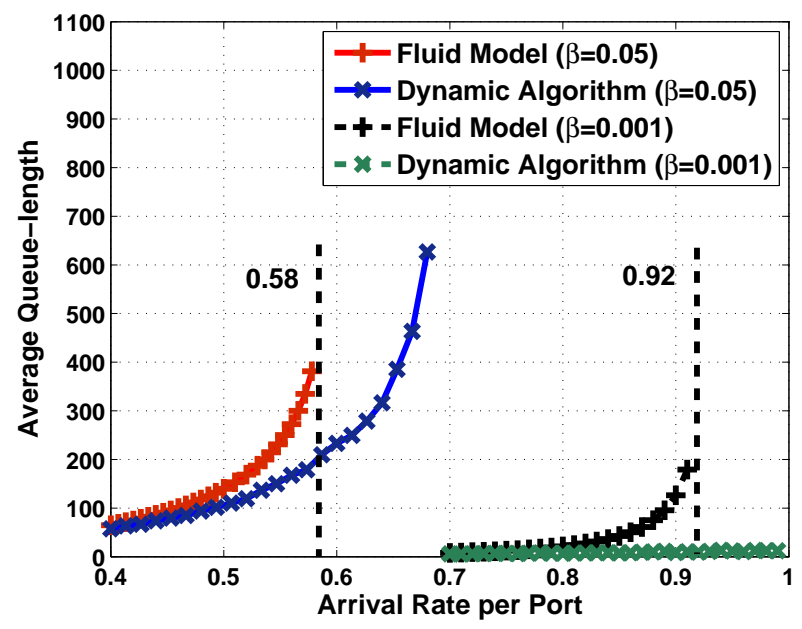

Figure 4: Equilibrium queue-lengths versus port arrival rate for the fluid model and the actual network implementing the backlog-based CSMA mechanism with AQM.

the achieved port throughput of the backlog-based CSMA policy with AQM as a function of the exogenous link arrival rate for $\beta=0.05$. We make two observations:

- First, we observe that the throughput increases with the arrival rate until the threshold level of 0.58 (cf. Figure 4) is reached. Beyond this point, the AQM rejects a higher percentage of the incoming packets in order to maintain the throughput level close to the capacity of 0.58. Thus, as desired, the throughput is kept at the level of system capacity even when the arrival rate exceeds the maximum stabilizable throughput level.

- Second, we observe that the increase in the arrival rate results in proportional increase in the throughput. The slope of the increase is smaller than one due to the presence of AQM which drops packets as the congestion level increases.

All of these simulations and numerical computations support the arguments and predictions of the previous sections. We see that, even under stochastic conditions, the backlog-based CSMA policy with AQM operates effectively to stabilize the system while achieving high throughput with a completely distributed.

\section{DISCUSSIONS AND CONCLUSIONS}

We present a fluid flow model to analyze backlog-based CSMA policies. The model is obtained using a CSMA fixed point approximation that has been recently proposed and analyzed. We provided numerical case studies to illustrate the accuracy of the fluid-flow model.

The analysis presented in this paper is restricted to a particular network topology, and the convergence result that we obtained assumes symmetric link arrival rates and that all queue-lengths are initially equal to 0 . Future work is to extend the analysis to general network topologies and arrival patterns. 


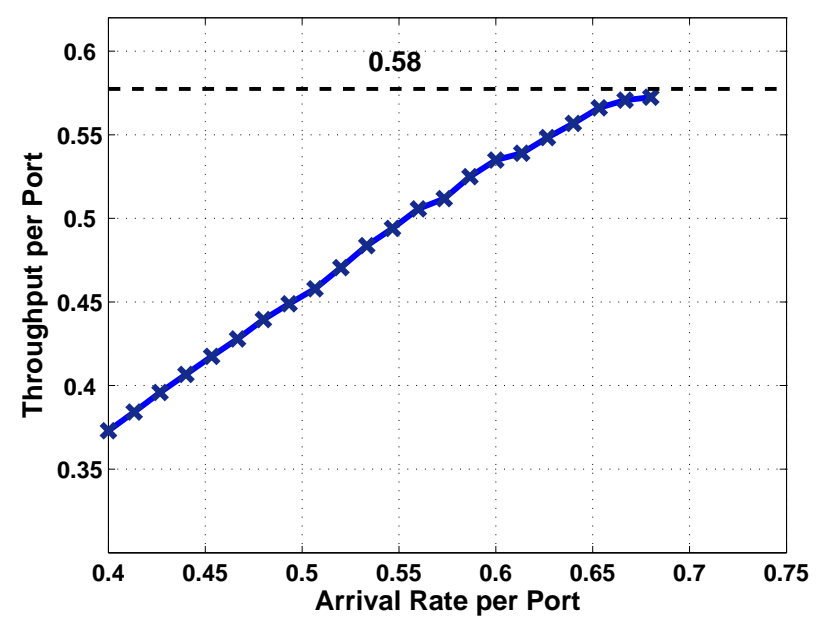

Figure 5: Achieved Throughput per port under the backlog-based CSMA policy with AQM versus arrival rate.

\section{REFERENCES}

[1] D. Bertsekas. Nonlinear Programming. Athena Scientific, Belmont, MA, 1999.

[2] D. Bertsekas and R. Gallager. Data Networks. Prentice Hall, Englewood Cliffs, NJ, 1990.

[3] A. Eryilmaz, A. Ozdaglar, and E. Modiano. Polynomial complexity algorithms for full utilization of multi-hop wireless networks, 2006. LIDS Technical Report.

[4] P. Gupta, Y. Sankarasubramaniam, and A. Stolyar. Random-access scheduling with service differentiation in wireless networks. In Proceedings of IEEE INFOCOM, 2005.

[5] L. Jiang and J. Walrand. A csma distributed algorithm for throughput and utility maximization in wireless networks. In Proceedings of Allerton Conference, Monticello, IL, 2008.

[6] K. Kar, S. Sarkar, and L. Tassiulas. Achieving proportionally fair rates using local information in aloha networks. In Proceedings of Allerton Conference, Monticello, IL, October 2003.

[7] X. Lin and S. Rasool. Constant-time distributed scheduling policies for ad hoc wireless networks. In Proceedings of $C D C, 2006$.

[8] X. Lin and N. Shroff. The impact of imperfect scheduling on cross-layer rate control in multihop wireless networks. In Proceedings of IEEE Infocom, Miami, FL, March 2005.

[9] P. Marbach. Rate control in random access networks: The finite node case. In Proceedings of IEEE Conference on Decision and Control, Paradise Island, Bahamas, December 2004.

[10] P. Marbach and A. Eryilmaz. A backlog-based csma mechanism to achieve fairness and throughput-optimality in wireless networks. In Proceedings of Allerton Conference, Monticello, IL, 2008.

[11] P. Marbach, A. Eryilmaz, and A. Ozdaglar. On the throughput-optimality of csma in wireless multihop networks. Computer Networks Research Lab, University of Toronto, Technical Report CNRL-08-002, August 2008.

[12] E. Modiano, D. Shah, and G. Zussman. Maximizing throughput in wireless networks via gossiping. In ACM SIGMETRICS/IFIP Performance, 2006.

[13] A. Proutiere, C. Bordanave, and D. M. Donald. Performance of random medium access - an asymptotic approach. In In Proceedings of Sigmetrics, Anapolis, MD, 2008.

[14] D. Shah and R. Sreevastsa. Reversible networks, distributed optimization, and network scheduling: what do they have in common? In Proceedings of CISS, Princeton, NJ, 2008.

[15] G. Sharma, R. Mazumdar, and N. Shroff. On the complexity of scheduling in multihop wireless systems. In Proceedings of MobiCom, Los Angeles, CA, September 2006

[16] L. Tassiulas and A. Ephremides. Stability properties of constrained queueing systems and scheduling policies for maximum throughput in multihop radio networks. IEEE Transactions on Automatic Control, 36:1936-1948, December 1992.

[17] X. Wu and R. Srikant. Regulated maximal matching: A distributed scheduling algorithm for multi-hop wireless networks with node-exclusive spectrum sharing. In Proceedings of IEEE Conference on Decision and Control., 2005.

[18] C. Yuen and P. Marbach. Price-based rate control in random access networks. IEEE/ACM Transactions on Networking, 13(5):1027-1040, October 2005.

\section{APPENDIX}

\section{A. PROOF OF PROPOSITION 10}

Note that for

$$
q_{(i, j)}=q, \quad(i, j) \in \mathcal{L},
$$

the CSMA fixed point equation of Eq. 9 is given by

$$
\rho(q)=\frac{\beta}{1+\beta-e^{-N \epsilon q \rho(q)}},
$$

where $q$ is the queue-length of the individual connections. We have the following result for the CSMA fixed point $\rho(q)$ of Eq. (14).

Lemma 2. The CSMA fixed point $\rho(q), q \geq 0$, of Eq. (14) is strictly decreasing in $q$ with $\rho(0)=1$ and $\lim _{q \rightarrow \infty} \rho(q)=$ $\frac{\beta}{1+\beta}$.

Proof. Using Eq. (14), we have $\rho(0)=1$ and $\rho(q) \in$ $[\beta /(\beta+1), 1]$ for $q \geq 0$. Let the function $f(q, \rho)$ be given by

$$
f(q, \rho)=\rho-\frac{\beta}{1+\beta-e^{-N \epsilon q \rho(q)}} .
$$

Note that $f(q, \rho(q))=0$. Using the implicit function theorem ([1]), for $q \geq 0$ we have

$$
\frac{d}{d q} \rho(q)=-\frac{\frac{\partial f}{\partial q} f(q, \rho(q))}{\frac{\partial f}{\partial \rho}(q, \rho(q))}=-\frac{\frac{\beta N \epsilon \rho(q) e^{-N \epsilon q \rho(q)}}{\left(1+\beta-e^{-N \epsilon q \rho(q)}\right)^{2}}}{1+\frac{\beta N \epsilon q e^{-N \epsilon q \rho(q)}}{\left(1+\beta-e^{-N \epsilon q \rho(q)}\right)^{2}}}<0,
$$


where we used that $\rho(q) \in[\beta /(\beta+1), 1]$ and $\rho(0)=1$. It follows that $\rho(q)$ is a strictly decreasing function of $q$. Finally, note that $\lim _{q \rightarrow \infty} f(q, \beta /(1+\beta))=0$ which implies that $\lim _{q \rightarrow \infty} \rho(q)=\beta /(1+\beta)$.

Let $G(q)=N \epsilon q \rho(q)$, where $\rho(q)$ is the above defined CSMA fixed point of Eq. (14).

Lemma 3. We have that $G(0)=0$ and $G(q)$ is strictly increasing in $q$ for $q \geq 0$ with $\lim _{q \rightarrow \infty} G(q)=\infty$.

Proof. From (14), we have $\rho(q)=\beta /\left(\beta+1-e^{-G(q)}\right)$. As $\rho(0)=1$, it follows that $G(0)=0$. By Lemma 2, $\rho(q)$ is strictly decreasing in $q$ and hence $G(q)$ is strictly increasing in $q$. Finally, as $\lim _{q \rightarrow \infty} \rho(q)=\beta /(\beta+1)$, it follows that $\lim _{q \rightarrow \infty} G(q)=\infty$.

\section{Proof of Proposition 1}

Proof. Let

$$
G^{*} \triangleq \min \left\{G \in\left[0, G^{+}(\beta)\right]: \tau(G) e^{-G}=N \lambda\right\} .
$$

Such a $G^{*}$ exists as $\tau(G) e^{-G}$ is continuous in $G$ on $\left[0, G^{+}(\beta)\right]$ and we have by assumption that $\tau(0) \leq N \lambda \leq \tau\left(G^{+}(\beta)\right) e^{-G^{+}(\beta)}$.

Note that the queue-length function $q(t)$ is continuous in $t$. Using the proof of Lemma 2 one can show that $\rho(q)$ is continuous in $q$ [11], and it follows that $G(t)=N \epsilon q(t) \rho(t)$ is continuous in $t$.

Suppose that Proposition 1 is not true, i.e. that $G(t)$ does not converge to $G^{*}$. We then distinguish the following two cases. First suppose that

$$
\lim \sup _{t \geq 0} G(t)>G^{*} .
$$

As $q(t=0)=0$, it follows from Lemma 3 that $G(t=0)=0$. Using the fact that $G(t)$ is continuous in $t$, it then follows that there exists a $t_{0}$ such that $G\left(t_{0}\right)=G^{*}$. However, in this case we have $\dot{q}\left(t_{0}\right)=0$ and $G(t)=G^{*}$ for all $t \geq t_{0}$. Hence, Eq. (15) cannot be true.

Next, suppose that

$$
\lim \sup _{t \geq 0} G(t)<G^{*} .
$$

In this case, we have that

$\dot{q}(t)=\lambda-T_{(i, j)}(\mathbf{p}(t))=\lambda-\frac{1}{N} \tau(G(t)) e^{-G(t)}>0, \quad t \geq 0$, and $q(t)$ and $G(t)$ are strictly increasing functions in $t$ for $t \geq 0$. As by assumption $G(t)$ is bounded by $G^{*}$, it follows that $G(t)$ converges to $G_{0}, G_{0}<G^{*}$. This implies that

$$
\dot{q}(t)=\lambda-T_{(i, j)}(\mathbf{p}(t)) \geq \lambda-\frac{1}{N} \tau\left(G_{0}\right) e^{-G_{0}}>0, \quad t \geq 0,
$$

and

$$
\lim _{t \rightarrow \infty} q(t)=\infty
$$

However, using Lemma 3, this contradicts the fact that $G(t)$ is bounded and Eq. (16) cannot hold. Thus, the proposition follows. 